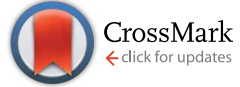

Cite this: RSC Adv., 2015, 5, 61021

\title{
Correlating flat band and onset potentials for solar water splitting on model hematite photoanodes $\dagger$
}

\author{
Beniamino landolo, t $^{\mathrm{a}}$ Haixiang Zhang, ${ }^{\mathrm{b}}$ Björn Wickman, ${ }^{\mathrm{a}}$ Igor Zorić, ${ }^{\mathrm{a}}$ Gavin Conibeer ${ }^{\mathrm{b}}$ \\ and Anders Hellman*a
}

\begin{abstract}
Hematite $\left(\alpha-\mathrm{Fe}_{2} \mathrm{O}_{3}\right)$ is a very promising material for solar water splitting that requires a high anodic potential to initiate the oxygen evolution reaction (OER). In this work, we explore the correlation between the downshift in flat band potential of hematite, $V_{\mathrm{fb}}$, and in onset potential of OER, $V_{\text {onset, caused by }}$ prolonged annealing. We observed a cathodic shift (i.e., towards lower potentials) of $200 \mathrm{mV}$ of $V_{\text {onset }}$ on model photoanodes consisting of ultra-thin hematite films, upon increasing the oxidation time during fabrication and without any further modifications. Detailed physical characterization, electrochemical impedance spectroscopy, and Mott-Schottky analysis revealed a quantitative correlation between the cathodic shift of $V_{\text {onset }}$ and a lowering of $V_{\mathrm{fb}}$. We identified a reduction in concentration of grain boundaries with increasing oxidation time, as the mechanism behind the observed shift of the $V_{\mathrm{fb}}$. The approach presented here can be seen as a complementary strategy to co-catalysts and other postfabrication treatments to lower $V_{\text {onset. }}$ Moreover, it is generically applicable to photoelectrodes used to carry out oxidation and reduction half-cell reactions.
\end{abstract}

Received 29th May 2015

Accepted 29th June 2015

DOI: 10.1039/c5ra10215d

www.rsc.org/advances operation in neutral and alkaline conditions; ${ }^{9}$ and (iii) its constituents are earth-abundant and non-toxic elements. ${ }^{10}$ Based on the value of $E_{\mathrm{G}}$ alone, a maximum theoretical solar to chemical energy conversion efficiency of $16.8 \%$ has been predicted for hematite based water oxidation. ${ }^{\mathbf{1 1}}$ However, the efficiency values achieved hitherto are at most of about $3 \% .^{12}$ This is mainly due to: (i) a low hole collection efficiency, particularly for energies close to the bandgap; ${ }^{\mathbf{1 3}}$ and (ii) a slow OER at the hematite/electrolyte interface, hence, requiring highly anodic potentials to achieve a reasonable rate. ${ }^{14}$

The typical energetics of a junction between hematite and an aqueous electrolyte is illustrated schematically in Fig. S1a. $\dagger$ As the materials brought into contact reach equilibrium, electrons transfer from hematite to the electrolyte will cause an upward band bending of the conduction and valence bands in the semiconductor. By applying an external bias to the semiconductor, the extent of the band bending can be increased, decreased and even reversed to be downwards. By definition, the flat band potential, $V_{\mathrm{fb}}$, is the electrochemical potential applied to the semiconductor that reduces this band bending to zero. Commonly found values of $V_{\mathrm{fb}}$ for hematite range between 0.4 and $0.8 \mathrm{~V} v s$. the reversible hydrogen electrode (RHE). ${ }^{15-18}$ For any potential more anodic than $V_{\mathrm{fb}}$, the upwards band bending drives photogenerated holes towards the solid/liquid interface. Then, if the quasi-Fermi energy level of the holes $E_{\mathrm{F}, \mathrm{p}}$ is lower than the OER potential, water oxidation can in principle take place. In practice, however, $E_{\mathrm{F}, \mathrm{p}}$ is higher than the top of the valence band due to a notably small value of photovoltage $\left(V_{\mathrm{ph}}\right)$ sustained by hematite. Also, as mentioned above,
Göteborg, Sweden. E-mail: anders.hellman@chalmers.se
Golied Physics, Chalmers University

${ }^{b}$ School of Photovoltaic and Renewable Energy Engineering, University of New South Wales, Sydney, NSW 2052, Australia

$\dagger$ Electronic supplementary information (ESI) available. See DOI: $10.1039 / \mathrm{c} 5 \mathrm{ra10215d}$

\$ Current address: Center for Electron Nanoscopy, Technical University of Denmark, DK-2800 Kgs. Lyngby, Denmark. 
the OER kinetics is slow. These two factors lead to the existence of a large (typically at least $400 \mathrm{mV}$ ) difference between $V_{\mathrm{fb}}$ and the experimentally observed $V_{\text {onset }}$. The quantity $V_{\text {onset }}-V_{\mathrm{fb}}$ is often referred to as overpotential for the OER. ${ }^{19}$

Fig. $\mathrm{S} 1 \mathrm{~b} \dagger$ illustrates the present gap between the performance of state-of-the-art hematite photoanodes and those of an ideal hematite photoanode. The latter delivers a photocurrent density (per projected unit area, $A_{\text {pro }}$ ) $j_{\text {max }}$ of $12 \mathrm{~mA} \mathrm{~cm}^{-2}$ at an electrochemical potential equal to or lower than $0.8 \mathrm{~V} v s$. RHE. ${ }^{20}$ The former photoanodes, instead, show an illuminated $V_{\text {onset }}$ of about $1.15 \mathrm{~V} v s$. RHE and reach $4 \mathrm{~mA} \mathrm{~cm}{ }^{-2}$ at $1.54 \mathrm{~V} v s$. RHE. ${ }^{21}$ Therefore, both enhancing $j_{\text {max }}$ and shifting $V_{\text {onset }}$ towards more cathodic potentials are needed to fully exploit the potential of hematite.

The issue of increasing $j_{\text {max }}$ is typically addressed by material doping $^{22-24}$ to increase conductivity and hence improve current collection, and/or nanostructuring to increase the surface to volume ratio. ${ }^{25-27}$ Most successful attempts to shift $V_{\text {onset }}$ cathodically can be grouped into three categories: ${ }^{28,29}$ (i) introducing OER co-catalysts, to accelerate the OER kinetics; ${ }^{25,30,31}$ (ii) depositing under- or overlayers of other oxides, to create homoor heterojunctions leading to reduced overpotential or to enhanced photovoltage ${ }^{32-35}$ (iii) post-fabrication treating the hematite surface. ${ }^{36-38}$ The aforementioned modifications of hematite result into either an increase of the $V_{\mathrm{ph}}$, or into a decrease of the overpotential (i.e., $V_{\text {onset }}-V_{\mathrm{fb}}$ ). A complementary approach to further shift $V_{\text {onset }}$ cathodically is to lower $V_{\mathrm{fb}}$. This will lead to a lowered $V_{\text {onset }}$ provided that worsening of other properties in the system, for instance slower OER kinetics or smaller $V_{\mathrm{ph}}$, does not overshadow this beneficial effect. Such a strategy is rarely investigated owing to the established concept that $V_{\mathrm{fb}}$ is an intrinsic property of the semiconductor given a certain fabrication procedure. Nevertheless, combining this strategy to others of proven efficiency is likely to be required to meet the goal of $12 \mathrm{~mA} \mathrm{~cm} \mathrm{~cm}^{-2}$ at $0.8 \mathrm{~V} v s$. RHE, especially considering that $j_{\max }$ is only achieved at a potential which is $0.3-$ $0.4 \mathrm{~V}$ more anodic than $V_{\text {onset }}$.

A systematic investigation of the correlation between the modification of $V_{\mathrm{fb}}$ and that of the photoelectrochemical performance of hematite photoanodes is missing in the literature, to the best of our knowledge, and we aim at correcting such deficiency in this work. Also, we address the following questions: can we shift the OER $V_{\text {onset }}$ on hematite by lowering $V_{\mathrm{fb}}$ without any further post-fabrication treatments, and without affecting negatively other PEC properties of the material? And if so, can we explain the observed trend(s) regarding $V_{\mathrm{fb}}$ and $V_{\text {onset }}$ ? Besides the intrinsic interest in deepening the understanding of hematite based water splitting, we anticipate that lowering $V_{\mathrm{fb}}$ of hematite, combined with strategies already established to shift $V_{\text {onset }}$ cathodically (and to enhance $j_{\text {max }}$ ), will help reaching the ideal performance of hematite photoanodes.

In order to address these questions, we employed a model system consisting of flat hematite films, and studied the impact of varying a key fabrication parameter, namely the oxidation time, on the PEC performance of the photoanodes. Our fabrication route (dry thermal oxidation of iron thin films) yields hematite photoanodes showing typical $j_{\max }$ between 0.1 and
$0.12 \mathrm{~mA} \mathrm{~cm}^{-2}$ (see results and discussion). This is commensurate with the $j_{\max }$ measured by other groups on flat hematite thin films in which the density of majority charge carriers is determined by oxygen vacancies in the lattice. ${ }^{16,18,24,39}$ We notice that these values of current per projected unit area $A_{\text {pro }}$ are more than an order of magnitude lower than those recorded on nanostructured, doped champion photoanodes. We mainly attribute such a difference to the fact that our samples are much flatter and therefore characterized by a smaller hematite/ electrolyte contact area $A_{\text {contact }}$ (for instance, the latter was determined to be 28 for nanocauliflower hematite fabricated by atmospheric pressure chemical vapor deposition ${ }^{25}$ ). Moreover, we stress that we focus here on $V_{\text {onset }}$ and $V_{\mathrm{fb}}$, rather than on $j_{\text {max }}$. Our samples are an excellent model system given the focus of this work, thanks to a less complicated interpretation of PEC data - in particular from electrochemical impedance spectroscopy, EIS - as compared to that obtained on nanostructured photoelectrodes. ${ }^{20} \mathrm{We}$ present samples with a range of values of $V_{\text {onset }}$, where the lowest ones are commensurate with those measured on the best hematite photoanodes without postfabrication treatments. We stress that it is important to analyze and understand the processes on all samples, with both high and low values of $V_{\text {onset }}$ in order to make models that can be extrapolated beyond state-of-the-art.

By prolonging the oxidation time of the films, and without any further treatment, we demonstrated a cathodic shift of $V_{\text {onset }}$ of about $200 \mathrm{mV}$. From EIS characterization, we obtained insights in the OER kinetics of the photoanodes and discriminated between losses taking places in the bulk of hematite and at the hematite/electrolyte interface. We used Mott-Schottky analysis to extract the $V_{\mathrm{fb}}$ in our films and thus compare the observed trends in $V_{\text {onset }}$ and $V_{\mathrm{fb}}$. Then, we employed a series of physical characterization techniques to investigate the physical mechanism(s) behind the observed PEC trends. We used scanning electron microscopy (SEM) and atomic force microscopy (AFM) to observe the morphology and topography of the hematite, respectively. We determined the oxidation state of $\mathrm{Fe}$ in the proximity of the hematite surface and investigated possible surface doping by means of X-ray photoemission spectroscopy (XPS). We measured the optical absorption coefficient and bandgap of hematite using optical spectroscopy. Finally, we used transmission electron microscopy (TEM) to qualitatively investigate the microstructure of hematite in terms of relative grain orientation and grain boundaries. We identified a reduction in concentration of grain boundaries upon increasing oxidation time as cause for the lowering of flat band potential.

\section{Methods}

\section{Fabrication}

Photoelectrodes were fabricated on indium tin oxide (ITO, PGO $\mathrm{GmbH}$, sheet resistance $\leq 20 \Omega$ per square) or fluorine doped tin oxide (FTO, Solaronix, sheet resistance $\leq 10 \Omega$ per square) covered glass substrates, according to a procedure described in detail elsewhere. ${ }^{40}$ Briefly, iron films were deposited on top of the respective substrate by physical vapor deposition (PVD 225, 
Kurt. J. Lesker, base pressure $<5 \times 10^{-7}$ mbar). Complete conversion into $\mathrm{Fe}_{2} \mathrm{O}_{3}$ was achieved with thermal oxidation at $350{ }^{\circ} \mathrm{C}$ in a mixed $\mathrm{N}_{2}: \mathrm{O}_{2}(3: 1)$ atmosphere. The temperature was ramped from room temperature up to $350{ }^{\circ} \mathrm{C}$ at a rate of $15{ }^{\circ} \mathrm{C} \min ^{-1}$, after which the temperature was kept constant at $350{ }^{\circ} \mathrm{C}$ for a time $t_{\mathrm{ox}}$, which was between 0 and $24 \mathrm{~h}$ depending on the sample. Then, the furnace was allowed to cool down to room temperature naturally.

\section{Characterization}

SEM of the as-prepared samples was performed in a Zeiss Supra $60 \mathrm{VP}$ microscope, using an acceleration voltage of $5 \mathrm{kV}$. The topography was investigated with a Bruker Dimension ICON AFM. Five $500 \times 500 \mathrm{~nm}$ scans were acquired in tapping mode for each sample, and the roughness for each scan was calculated after image flattening using NanoScope Analysis v1.40r1 software (Bruker). The thickness of the films was measured with a Dektak 150 surface profiler (Veeco). The optical absorption in the films deposited on ITO was measured with a Cary 5000 spectrophotometer (Varian) equipped with the External Diffuse Reflectance Accessory (DRA) 2500, which accounts for optical reflection. The absorption in hematite was determined by using a blank ITO substrate as reference. The latter was exposed to the same furnace treatment as the hematite covered substrates. The absorption coefficient $\alpha$ was extracted from the measured absorption assuming a Lambertian absorption profile. The values obtained were used for Tauc analysis according to the formula $(\alpha h \nu)^{0.5}=\left(h \nu-E_{\mathrm{G}}\right)$, where $h \nu$ is the photon energy, $E_{\mathrm{G}}$ is the bandgap energy of the semiconductor and the exponent 0.5 indicates an indirect (i.e., phonon assisted) transition. The values of $E_{\mathrm{G}}$ for different samples were obtained by extrapolating to the horizontal axis the linear part of the curves in the Tauc plots in the energy range between 2.1 and $2.3 \mathrm{eV}$. XPS spectra were acquired in a Perkin Elmer Phi 5500 setup (base pressure $<10^{-10}$ mbar) using $\mathrm{Al}_{\mathrm{K} \alpha}$ radiation of $1.4866 \mathrm{keV}$. The XPS spectra were shifted using the $\mathrm{C}(1 \mathrm{~s})$ peak corresponding to adventitious carbon $(284.5 \mathrm{eV})$ as a reference. TEM was performed with a field emission Jeol JEM 2100F microscope at $200 \mathrm{kV}$. To facilitate the TEM analysis, thin silicon nitride membranes ('TEM windows'41) were used as support and hematite films were fabricated on the membranes using the same procedure as described above.

\section{Photoelectrochemical measurements}

All PEC characterization of the ITO/hematite samples was carried out in a Teflon based cell (Accent PN4300), using an Autolab PGSTAT302N potentiostat. A three electrodes configuration was used, with the ITO/hematite as working electrode, a $\mathrm{Pt}$ wire as counter-electrode and a $\mathrm{Ag} / \mathrm{AgCl}$ ( $\mathrm{KCl}$ saturated) reference electrode. A $0.1 \mathrm{M} \mathrm{KOH}$ solution $(\mathrm{pH}=12.9)$ was used as the electrolyte, and the working electrode was illuminated from the front in all measurements. The $\mathrm{Ag} / \mathrm{AgCl}$ electrode potential, $V_{\mathrm{Ag} / \mathrm{AgCl}}$, was converted to the reversible hydrogen electrode, $V_{\mathrm{RHE}}$, using the equation $V_{\mathrm{RHE}}=V_{\mathrm{Ag} / \mathrm{AgCl}}+V_{\mathrm{Ag} / \mathrm{AgCl}}^{0}+$ $0.059 \times \mathrm{pH}$, where $V_{\mathrm{Ag} / \mathrm{AgCl}}^{0}=0.197 \mathrm{~V}$ at $25^{\circ} \mathrm{C}$. The light source was a $300 \mathrm{~W}$ Xe lamp (Cermax_LX_300) equipped with a UV filter (420 nm cut-off wavelength, Schott), positioned at a distance of $38.5 \mathrm{~cm}$ from the PEC cell. At such a distance, the total light intensity impinging on the working electrode was estimated from product specification to be about the same as AM 1.5 filtered illumination provided by a solar simulator $\left(100 \mathrm{~mW} \mathrm{~cm}^{-2}\right)$, although with a different spectral distribution. Cyclic voltammetry was performed between 0.76 and $1.76 \mathrm{~V} v s$. RHE at a scan rate of $10 \mathrm{mV} \mathrm{s}^{-1}$. To obtain the photocurrent density $j$, the current was divided by the projected area in contact with the electrolyte, $A_{\text {pro }}$. Only the forward scan from cathodic to anodic potentials was plotted for ease of visualization. For the EIS measurements, the DC voltage was changed in steps of $0.1 \mathrm{~V}$ from 1.76 to $0.86 \mathrm{~V} v s$. RHE, on top of which an AC voltage of root mean square amplitude of $10 \mathrm{mV}$ and frequency varying between $10^{5}$ and $0.1 \mathrm{~Hz}$ was superimposed. Nyquist plots obtained under illumination were fitted using the software Nova v. 1.9 (Metrohm Autolab). Mott-Schottky analysis was performed in the dark, in the same potential window, using an AC voltage with a frequency of $10^{4} \mathrm{~Hz}$. Cyclic voltammetry of the FTO/hematite samples was performed in the same 3-electrode configuration, using a Gamry ref. 600 potentiostat, in a H-type glass cell with working electrode and counter-electrode compartments separated by a glass frit. The working electrode compartment had flat optical windows to allow for the illumination of the samples. A solar simulator (SKU SS150, Sciencetech. Inc.) was the illumination source, with intensity at the working electrode surface adjusted to $100 \mathrm{~mW} \mathrm{~cm}^{-2}$ using a NIST calibrated photodiode.

\section{Results and Discussion}

In light assisted OER from water splitting, as explained above, two quantities are of key interest, namely the onset potential $V_{\text {onset }}$ and the plateau photocurrent $j_{\text {max }}$ measured before purely electrochemical water splitting is observed. Fig. 1 shows the results of the cyclic voltammetry measurements performed on samples with different $t_{\mathrm{ox}}$ under illumination and in the dark. While samples with $t_{\mathrm{ox}}$ between 0 and $12 \mathrm{~h}$ show a maximum photocurrent between 0.11 and $0.12 \mathrm{~mA} \mathrm{~cm} \mathrm{~cm}^{-2}$, those with longer $t_{\mathrm{ox}}$ show negligible photoactivity. This is most likely due to the loss of electrical conductivity of ITO when exposed at temperatures of $350{ }^{\circ} \mathrm{C}$ for prolonged times. ${ }^{42}$ Therefore, in the remainder of the manuscript we will focus on samples with $t_{\mathrm{ox}} \leq 12 \mathrm{~h}$.

The maximum photocurrent $j_{\max }$ does not depend significantly on $t_{\mathrm{ox}}$ for potentials more anodic than $1.6 \mathrm{~V} v s$. RHE. As discussed in the introduction, the values $j_{\max }$ measured here are more than an order of magnitude lower than those measured on state-of-the-art photoanodes, however commensurate to those observed on photoanodes fabricated using similar routes. ${ }^{39}$ It is worth mentioning that we found an increase of $j_{\max }$ of about $20 \%$ as compared to hematite films fabricated via the same procedure and characterized in previous work. ${ }^{43}$ This difference is most likely caused by the differences in the spectral distribution of the illumination source. We recall that here the light intensity impinging on the sample was $100 \mathrm{~mW} \mathrm{~cm}^{-2}$, but not with an AM 1.5 spectral distribution. 

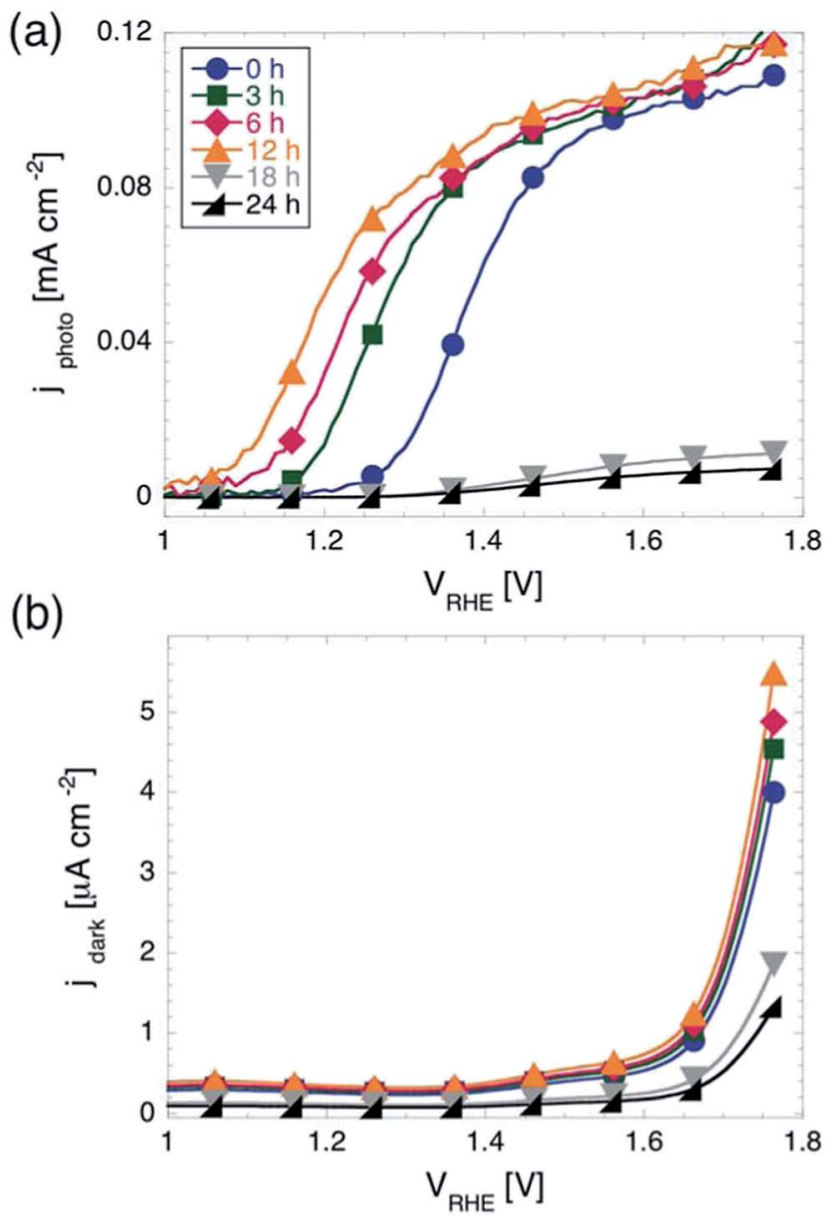

Fig. 1 Current density $j$ as function of electrochemical potential $V$ for hematite films on ITO. (a) Photocurrent density under UV-filtered illumination from a Xe-lamp. The onset potential for the OER $V_{\text {onset }}$ shifts of $200 \mathrm{mV}$ towards more cathodic potentials for $t_{\mathrm{ox}}$ increasing from 0 to $12 \mathrm{~h}$. For longer $t_{\mathrm{ox}}$, the films show negligible photoactivity. (b) Current density measured in the dark. The onset potential shifts only by around $30 \mathrm{mV}$ between $t_{\mathrm{ox}}=0 \mathrm{~h}$ and $t_{\mathrm{ox}}=12 \mathrm{~h}$.

Although all samples shown in Fig. 1a reach very similar values of $j_{\max }$ for high anodic potentials, they show a striking difference in $V_{\text {onset }}$, which moves towards more cathodic potentials for increasing $t_{\mathrm{ox}}$. Considering the quantitative determination of $V_{\text {onset }}$, different definitions can be found in the literature. For example, $V_{\text {onset }}$ has been defined as: (i) the potential at which the photocurrent exceeds a certain defined value $^{30}$ (ii) the potential at which $\mathrm{d} J / \mathrm{d} V$ exceeds a certain defined value, ${ }^{44}$ or (iii) the potential at which the tangent to the $J(V)$ curve in the region of maximum slope intersects the $J(V)$ curve measured in the dark. ${ }^{33}$ It should be noted that all these definitions are somewhat arbitrary and may be more or less appropriate depending on the shape of the $J(V)$ curves at hand. For instance, using method (iii) can result in a situation where a curve with a steep slope will have a more anodic $V_{\text {onset }}$ than one with a flatter slope, although they may have the same $V_{\text {onset }}$ according to methods (i) and/or (ii). We note that the $J(V)$ curves presented here show a very similar slope after the photocurrent starts rising. Therefore, although the choice of method to define
$V_{\text {onset }}$ might slightly affect the exact values extracted from the $J(V)$ curves, the shifts in $V_{\text {onset }}$ with varying $t_{\text {ox }}$ will be practically be independent of the chosen method. Here we used method (i), with $10 \mu \mathrm{A} \mathrm{cm} ~_{-2}$ as a threshold in photocurrent density (corresponding to about $10 \%$ of $j_{\max }$ ). According to this definition, we found $V_{\text {onset }}$ to be equal to $1.28 \mathrm{~V} v s$. RHE for $t_{\mathrm{ox}}=0$. Upon increasing $t_{\mathrm{ox}}$ from to 3,6 , and 12 hours, $V_{\text {onset }}$ shifts significantly towards more cathodic potentials, to $1.19,1.13$, and 1.08 $\mathrm{V} v \boldsymbol{~ s . ~ R H E , ~ r e s p e c t i v e l y . ~}$

The onset potential showed a much weaker dependency on $t_{\mathrm{ox}}$ for cyclic voltammetry in the dark, as shown in Fig. 1b. Using method (i) again (with threshold current corresponding to $10 \%$ of the maximum current observed in the dark in the potential window investigated), we found that the onset potential shifts only $30 \mathrm{mV}$ in total when increasing $t_{\mathrm{ox}}$ from 0 to $12 \mathrm{~h}$. This indicates that the effect of prolonging $t_{\mathrm{ox}}$ on the OER in the dark is negligible.

We further investigated the PEC properties of the photoanodes via EIS. Recent studies have established EIS as a powerful technique to characterize the dynamics of charge transfer at the hematite/electrolyte interface, as well as of charge transport in the bulk of the semiconductor. ${ }^{\mathbf{1 4 , 4 5}}$ Data from EIS measurements is often shown in form of Nyquist plots, where the real component and negative imaginary component of the complex impedance $Z$ are plotted on the horizontal and vertical axis, respectively. Fig. 2a shows the Nyquist plots obtained for the $6 \mathrm{~h}$ sample, under illumination, at some relevant values of DC bias. A qualitative decrease in total impedance is visible with increasing potential (at $1.16 \mathrm{~V}$ vs. RHE the impedance values at lower frequencies are well outside the range of impedance in the plot). The presence of two semicircles indicates that two time constants are present in the system. We found the same evolution of the Nyquist plots for increasingly anodic potential for all samples measured.

In order to correlate changes in the Nyquist plots with changes in the properties of charge transport through the photoanode and of charge transfer at the hematite/ semiconductor interface, equivalent circuit modeling is required. For photoelectrodes where water oxidation performance is hampered by slow OER kinetics, an appropriate circuit is shown in the inset of Fig. 2a, which has been discussed and employed previously for studying hematite photoanodes. ${ }^{\mathbf{8 , 4 5}}$ In brief, the electrical components of this equivalent circuit are: $R_{\mathrm{S}}$, the contact resistance between ITO and hematite; $C_{\mathrm{bulk}}$, the capacitance originating from the charge in the space-charge layer in hematite; $R_{\text {trap }}$, the resistance associated with transport of charge through the bulk of hematite; $C_{\mathrm{SS}}$, the chemical capacitance associated with surface states at the hematite/ electrolyte interface, ${ }^{46}$ and $R_{\mathrm{SS}}$, the resistance associated with charge transfer from hematite to the electrolyte. Within this model, the high and low frequency semicircles in the Nyquist plots are associated with bulk and surface properties of the photoanode, respectively. It is clear that the impedance associated with the low frequency semicircle decrease considerably with increasing potential, while the impedance associated with high frequency is basically independent of the applied bias. 

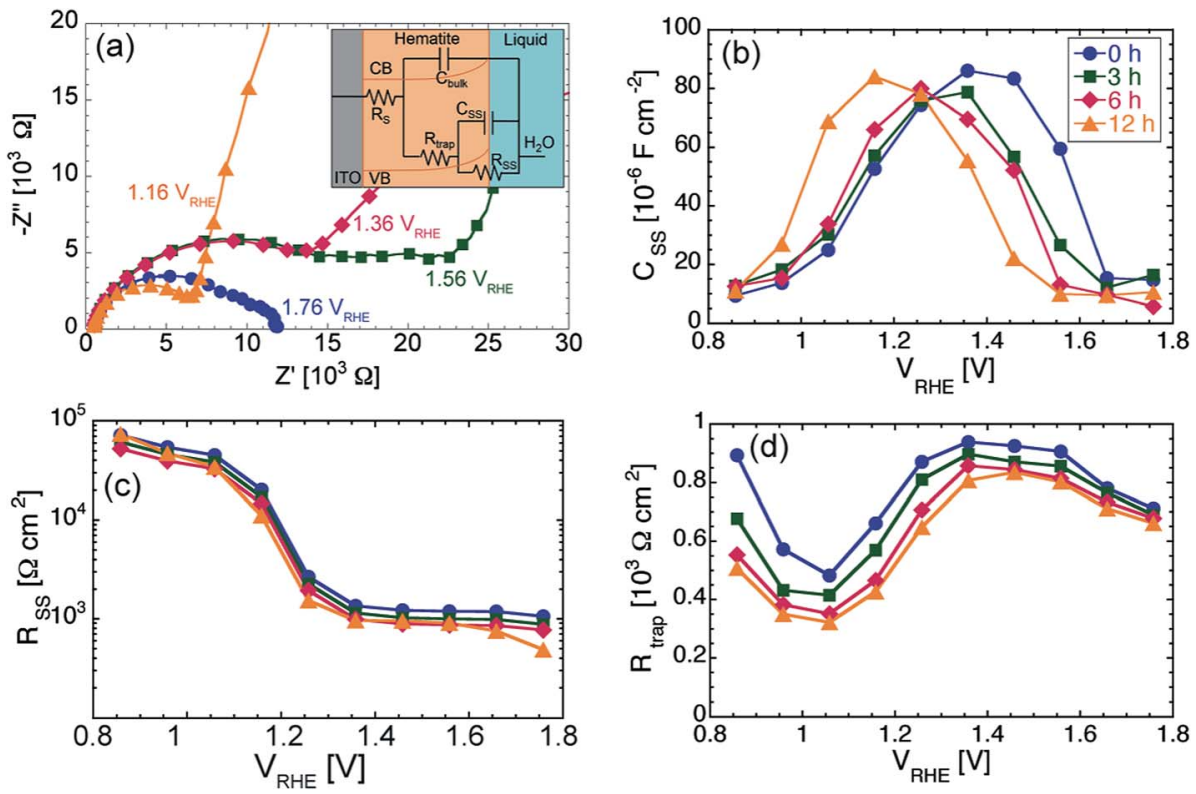

Fig. 2 (a) Representative Nyquist plots obtained from EIS measurements on the $t_{\mathrm{ox}}=6 \mathrm{~h}$ sample under illumination at different applied potential. Inset: equivalent circuit used to fit the Nyquist plots under illumination. (b-d) Results from the equivalent circuit fitting of the Nyquist plots. The following quantities are plotted as function of applied potential: (b) Surface states capacitance $C_{\mathrm{SS}}$ (c) Charge transfer resistance $R_{\mathrm{SS}}$ (d) trapping resistance $R_{\text {trap }}$.

By fitting of the Nyquist plots using the equivalent circuit described above, we extracted the values of $C_{\mathrm{SS}}, R_{\mathrm{trap}}$ and $R_{\mathrm{SS}}$ for all samples as function of the applied DC potential. The results are shown in Fig. 2b-d. For each sample, $C_{\mathrm{Ss}}(V)$ first increases for more anodic potential, reaches a maximum and then decreases back to about its initial value for the most anodic potentials. Moreover, the potential at which $C_{\mathrm{ss}}(V)$ peaks is close to the onset potential $V_{\text {onset }}$. This fact is a signature that the OER under illumination takes place via surface states for potential values close to $V_{\text {onset }}$. These surface states are first charged and then discharged when the potential is sufficiently anodic to start the OER. We estimated the charge density on the surface state $Q_{\mathrm{Ss}}$ by integrating $C_{\mathrm{SS}}$ with respect to $V$. We found $Q_{\text {ss }}$ to be between 38.2 and $39.2 \mu \mathrm{C} \mathrm{cm}^{-2}$ for the $12 \mathrm{~h}$ and $0 \mathrm{~h}$ samples, respectively. In other words $Q_{\mathrm{Ss}}$ is basically constant regardless of $t_{\mathrm{ox}}$. These observations, together with the weak dependency of the dark electrolysis onset potential on $t_{\mathrm{ox}}$, point to very similar OER kinetics for all samples.

The hematite/electrolyte charge transfer resistance, $R_{\mathrm{SS}}(V)$, is plotted in Fig. 2c, from which we see that the transfer of holes from the semiconductor to the electrolyte is not significantly affected by changing $t_{\mathrm{ox}}$. Fig. $2 \mathrm{~d}$ shows that the resistance associated with recombination of charge carriers in the bulk of the hematite, $R_{\text {trap }}(V)$, has a similar behavior for all samples, with a minimum at $1.06 \mathrm{~V} v s$. RHE and a flatter region for potentials more anodic than $1.36 \mathrm{~V} v s$. RHE. However, the values of $R_{\text {trap }}$ are clearly decreasing for increasing $t_{\mathrm{ox}}$, with the strongest effect for potentials more cathodic than $1.56 \mathrm{~V} v s$. RHE.

We can summarize the main findings of our equivalent circuit analysis as follows: (1) the results from the analysis of the $C_{\mathrm{SS}}(V)$ and $R_{\mathrm{SS}}(V)$ plots are additional evidence that varying $t_{\mathrm{ox}}$ does not affect the OER kinetics under illumination; (2) the decrease of $R_{\text {trap }}(V)$ with increasing $t_{\mathrm{ox}}$ for low anodic bias indicates a possible beneficial effect of prolonged oxidation time on the charge carrier transport within the films.

Mott-Schottky (MS) analysis is commonly performed in the dark, in order to extract not only $V_{\mathrm{fb}}$, but also the donor/acceptor (for $\mathrm{n}$ - and p-type semiconductor, respectively) concentration, $N_{\mathrm{D}}$. For a perfectly planar semiconductor-electrolyte interface (i.e. where $\left.A_{\text {pro }}=A_{\text {contact }}\right)$, the MS equation reads $\left(A_{\text {pro }} / C_{\text {bulk }}\right)^{2}=$ $2\left(V-V_{\mathrm{fb}}-k_{\mathrm{b}} T / e\right) /\left(\varepsilon_{0} \varepsilon_{\mathrm{r}} N_{\mathrm{D}}\right)$, where, $C_{\mathrm{bulk}}$ is the capacitance of the space-charge layer, $k_{\mathrm{b}}$ is the Boltzmann constant, $T$ is the absolute temperature, $e$ is the elementary charge, $\varepsilon_{0}$ is the permittivity of vacuum and $\varepsilon_{\mathrm{r}}$ is the relative permittivity of the semiconductor. ${ }^{47}$ The validity of this simple relationship between $C_{\text {bulk }}$ and $V-V_{\mathrm{fb}}$ lies on the following assumptions: (i) $C_{\text {bulk }}$ is much smaller than the capacitance $C_{\mathrm{H}}$ associated with the Helmholtz layer in the electrolyte; (ii) the donors/acceptors are uniformly distributed in space, have all the same energy, and are all ionized; (iii) the width of the space-charge layer $W_{\mathrm{SC}}$ is much smaller than the thickness $d$ of the semiconductor. Under these assumptions, the MS plot is linear, and $N_{\mathrm{D}}$ can be obtained from the relationship $N_{\mathrm{D}}=2 / \varepsilon_{0} \varepsilon_{\mathrm{r}} e S$, where $S$ is the slope of the plot, while $V_{\mathrm{fb}}$ is obtained from the intercept with the horizontal axis.

The MS plots for the values of $t_{\mathrm{ox}}$ investigated in this work are indeed linear for potentials between 0.86 and $1.36 \mathrm{~V} v$ s. RHE, as shown in Fig. 3a, while for potentials more anodic they show a concave shape. The latter could in principle be explained by assuming a special non-uniform distribution of ionized donors..$^{48}$ In particular, one would have to assume a continuously increasing $N_{\mathrm{D}}$ with increasing distance from the hematite/ electrolyte interface. However, we do not consider such a 
(a)

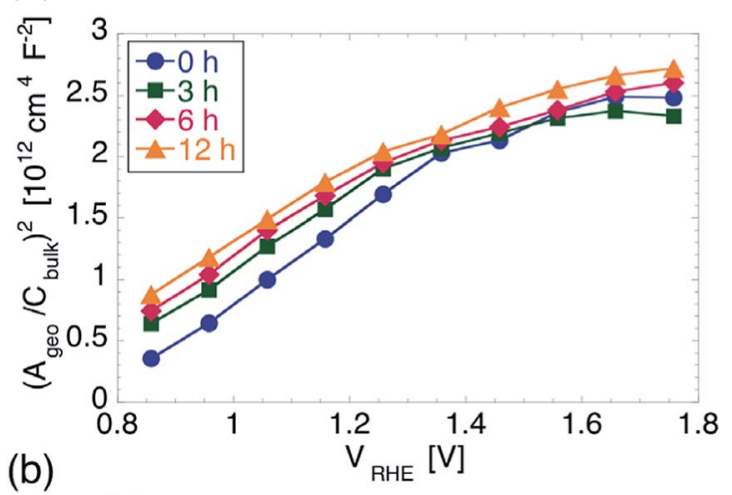

(b)

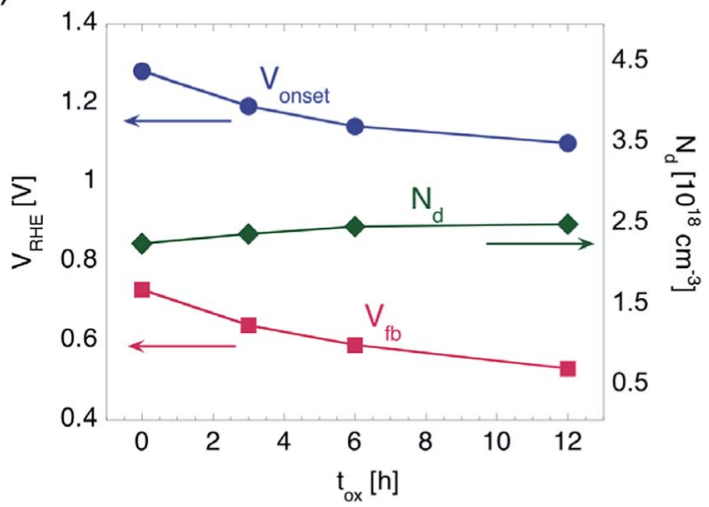

Fig. 3 (a) Mott-Schottky plots for hematite films on ITO. (b) Onset potential $V_{\text {onset }}$ and flat band potential in hematite $V_{\text {fb }}$ (left $y$-axis) and donor concentration $N_{\mathrm{D}}$ (right $y$-axis) as function of $t_{\mathrm{ox}}$.

possibility as plausible, since the oxidation of $\mathrm{Fe}$ into $\mathrm{Fe}_{2} \mathrm{O}_{3}$ is carried out for a sufficiently long time (including ramp up and cool down time) for the resulting films to have uniform electronic properties throughout their thickness.

Instead, the considerably small thickness of our hematite films makes the approximation $W_{\mathrm{SC}}<d$ questionable. It has been shown that, in photoanodes consisting of a thin $\mathrm{TiO}_{2}$ deposited on ITO, the depletion region can extend into the ITO for sufficiently high anodic potentials, and the MS equation needs to be modified to take this effect into account. ${ }^{49}$ In particular, the MS plot was predicted to have two distinct slopes, one at more cathodic potentials, which is determined by the $\varepsilon_{\mathrm{r}}$ and $N_{\mathrm{D}}$ of the $\mathrm{TiO}_{2}$, and one for more anodic potentials, which is determined by the $\varepsilon_{\mathrm{r}}$ and $N_{\mathrm{D}}$ of the ITO. The potential $V_{\mathrm{th}}$ where the slope changes is the potential at which $W_{\mathrm{SC}}$ in the $\mathrm{TiO}_{2}$ equals the $\mathrm{TiO}_{2}$ thickness. The following relationship between $d$ and $V_{\text {th }}$ was then found: $d=\varepsilon_{0} \varepsilon_{\mathrm{r}} S^{0.5}\left(V_{\mathrm{th}}-V k T / e\right)^{0.5}$. We followed a similar approach to further analyze our MS plots. We note that the potentials applied here were not so anodic that the second slope could be determined without a large deviation. Therefore we used, for the slope at high anodic potentials, the values of $\varepsilon_{\mathrm{r}}$ (ITO) $=9$ and $N_{\mathrm{D}}$ (ITO) $=10^{20} \mathrm{~cm}^{-3}$, which are normally found for ITO films with similar sheet resistance as compared to the ones used here. ${ }^{49,50}$ Once $V_{\text {th }}$ is determined and $d$ is known, $\varepsilon_{\mathrm{r}}$ can be extracted. We found that $\varepsilon_{\mathrm{r}}$ only varies between 18.9 and 19 when increasing $t_{\mathrm{ox}}$. We note that these values for $\varepsilon_{\mathrm{r}}$ are considerably smaller than the values of 32 and 80 often plugged into the simplest form of the MS equation to directly extract $N_{\mathrm{D}}{ }^{28,51}$ Within the approach used here, instead, we can use the $\varepsilon_{\mathrm{r}}$ extracted from our MS plots to obtain $N_{\mathrm{D}}$. We found the latter to increase from $2.28 \times 10^{18} \mathrm{~cm}^{-3}$ to $2.52 \times 10^{18} \mathrm{~cm}^{-3}$ with $t_{\mathrm{ox}}$ increasing from 0 to $12 \mathrm{~h}$. From the measurements performed here we cannot determine unambiguously the origin of such difference in $N_{\mathrm{D}}$. Nevertheless, we stress that the increase of $N_{\mathrm{D}}$ is only of a factor of 1.1, which we judge insufficient to explain the considerable $V_{\text {onset }}$ shift found from cyclic voltammetry. Moreover, we found a quantitative correlation between the shift of $V_{\text {onset }}$ and that of $V_{\mathrm{fb}}$, as explained below.

In order to evaluate $V_{\mathrm{fb}}$, we fitted the linear part of the MS plots, where only the properties of hematite come into play. Since we did not measure directly $A_{\text {contact }}$, we used the value of $A_{\text {pro }}\left(0.1 \mathrm{~cm}^{2}\right)$ as contact area for all samples, and we found $V_{\mathrm{fb}}$ to shift from $0.73 \mathrm{~V}$ to $0.53 \mathrm{~V} v s$. RHE. Such a shift towards more negative potentials follows closely the shift of $V_{\text {onset }}$ observed during the cyclic voltammetry measurements, as illustrated in Fig. 3b. The difference between $V_{\text {onset }}$ and $V_{\mathrm{fb}}$ is about $550 \mathrm{mV}$ irrespective of $t_{\mathrm{ox}}$, which points once again to very similar OER kinetics for all samples. This is in contrast to the reduction in $V_{\text {onset }}$ obtained upon employing electrocatalysts or other surface coatings, which do not change $V_{\mathrm{fb}}$ appreciably. ${ }^{30,52}$ The shift of $V_{\mathrm{fb}}$ could in principle be caused by a decrease in the Helmholtz layer capacitance. A treatment of the solid/liquid interface that includes explicitly $C_{\mathrm{H}}$ has shown to predict a MS plot with the same slope as in the simplest case, ${ }^{53}$ but with an intercept shifted by an amount equal to $\varepsilon_{0} \varepsilon_{\mathrm{r}} e N_{\mathrm{D}} / 2 C_{\mathrm{H}}{ }^{2}=1 / S C_{\mathrm{H}}{ }^{2}$. In order to obtain the extracted $200 \mathrm{mV}$ shift of $V_{\mathrm{fb}}$, one would have to assume an increase of $C_{\mathrm{H}}$ of a factor of 25 upon increasing $t_{\mathrm{ox}}$ from 0 to $12 \mathrm{~h}$, which we judge unreasonable given the similarity of the hematite/electrolyte interface properties as extracted by EIS under illumination.

Another possible mechanism of the observed shift of $V_{\mathrm{fb}}$ could be the introduction of In or Sn atoms from ITO into the hematite, which would be consistent with the slight increase of $N_{\text {D }}$ extracted previously. The modification of $V_{\mathrm{fb}}$ upon doping has been observed, for instance, in other semiconductors used in PEC cells/solar cells. ${ }^{54,55}$ However, direct evidence that neither In nor Sn did not diffuse from ITO to the surface of the photoanodes is offered by XPS measurements, as discussed further on. Furthermore, we performed control cyclic voltammetry measurements on hematite samples deposited FTO, which is known to be thermally stable up to $700{ }^{\circ} \mathrm{C}$. The photocurrent measurements carried out on the FTO based films demonstrated a similar cathodic shift of the onset potential upon increasing $t_{\mathrm{ox}}$ from 3 to $12 \mathrm{~h}$ to that obtained for the films deposited on ITO, which confirms that the observed $V_{\text {onset }}$ is also independent of the electron collection layer underneath hematite.

Thanks to the MS analysis, we established a quantitative correlation between the extracted shift of $V_{\mathrm{fb}}$ and the shift of $V_{\text {onset }}$ observed during the cyclic voltammetry measurements. While $V_{\mathrm{fb}}$ is typically determined by the properties of the solid/ liquid junction and of the space-charge layer, $V_{\text {onset }}$ is determined by the properties of the whole photoelectrode. However, due to the very small thickness of the films used here, it is 
reasonable to consider both $V_{\mathrm{fb}}$ and $V_{\text {onset }}$ as determined by the properties of the whole film. Nevertheless, it is not clear at this stage if the shift of $V_{\mathrm{fb}}$ is correlated to any change in the physical properties of the hematite films, which we characterized in deeper detail.

We measured the hematite thickness by surface profiler, and found it to be $25 \pm 2 \mathrm{~nm}$ irrespective of $t_{\mathrm{ox}}$. SEM inspection did not indicate an obvious difference in morphology between the samples, as shown in Fig. S2.† Investigation by AFM led to the conclusion that the topography of the samples is not affected appreciably by prolonging $t_{\mathrm{ox}}$, as shown in Fig. S3. $\uparrow$ The root mean square roughness, $R_{\mathrm{q}}$, was found to vary only very slightly from $2.3 \pm 0.15 \mathrm{~nm}$ for $t_{\mathrm{ox}}=0 \mathrm{~h}$ to $2.32 \pm 0.18 \mathrm{~nm}$ for $t_{\mathrm{ox}}=12 \mathrm{~h}$, which justifies the use of the same value of contact area for all the samples.

We carried out XPS measurements on sample with $t_{\mathrm{ox}}$ equal to 0 and 12 , in order to perform analysis of chemical composition and electronic states of the surface of the films, and the results are summarized in Fig. 4. First, we notice that no peaks appear in the survey scans (panel a) at energies corresponding to peaks indicating the presence of In or Sn, which is a further, direct confirmation that neither In nor Sn did not diffuse up to the hematite surface. Second, the normalized XPS Fe(2p) and $\mathrm{O}(1 \mathrm{~s})$ signals (panels $\mathrm{b}$ and $\mathrm{c}$ respectively) are practically identical in shape, indicating that surface composition of the sample and oxidation state of the iron within a few $\mathrm{nm}$ from the surface are not affected by changing $t_{\mathrm{ox}}$.

We characterized the optical properties of the films using spectrophotometry, and the results are summarized in Fig. 5. It is worth mentioning that the use of the integrating sphere accessory to perform these measurements enabled us to directly measure the sum of scattered (including reflected) light and transmitted light, from which we obtained the absorption Abs = $1-(\mathrm{Sca}+\mathrm{Tr})$. The onset of optical absorption between 630 and $600 \mathrm{~nm}$ indicates that the oxidation process is complete even for $t_{\mathrm{ox}}=0 \mathrm{~h}$, i.e. when ramping up the temperature to $350{ }^{\circ} \mathrm{C}$ and starting the cool-down immediately afterwards. The absorption profile of the $0 \mathrm{~h}$ sample, however, differs slightly from the other spectra. In particular, the absorption is lower for wavelengths between 420 and $560 \mathrm{~nm}$, whereas it is slightly higher between

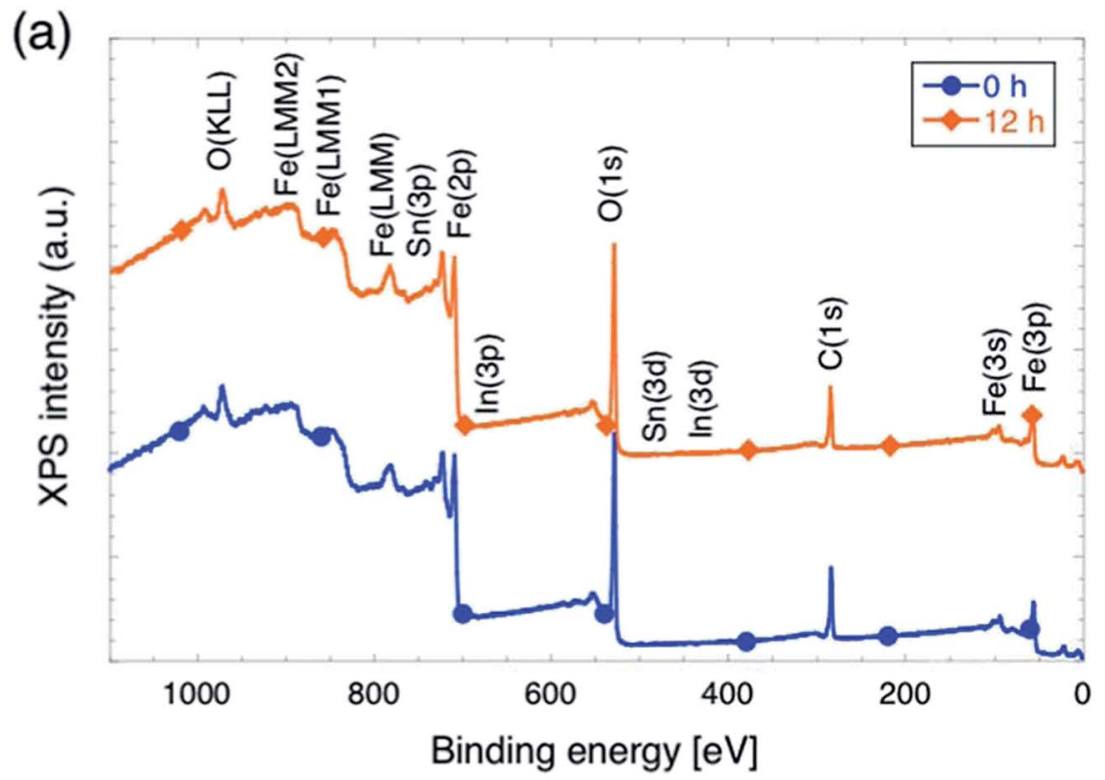

(b)

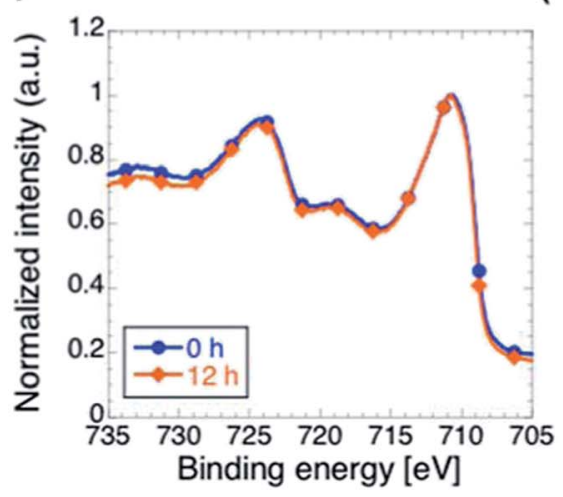

(c)

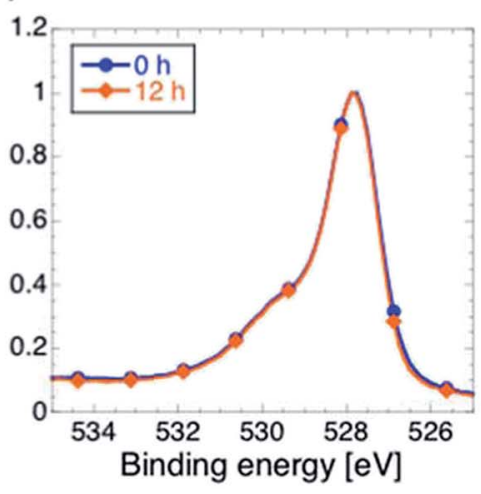

Fig. 4 XPS characterization for hematite films on ITO with $t_{\mathrm{ox}}=0 \mathrm{~h}$ and $t_{\mathrm{ox}}=12 \mathrm{~h}$. (a) Survey scans with peaks identification. The curves are shifted on the vertical axis for ease of visualization. Clearly, no In or Sn is detected. (b). Normalized intensity profiles of Fe(2p) peaks. (c) Normalized intensity profiles of $\mathrm{O}(1 \mathrm{~s})$ peaks. 


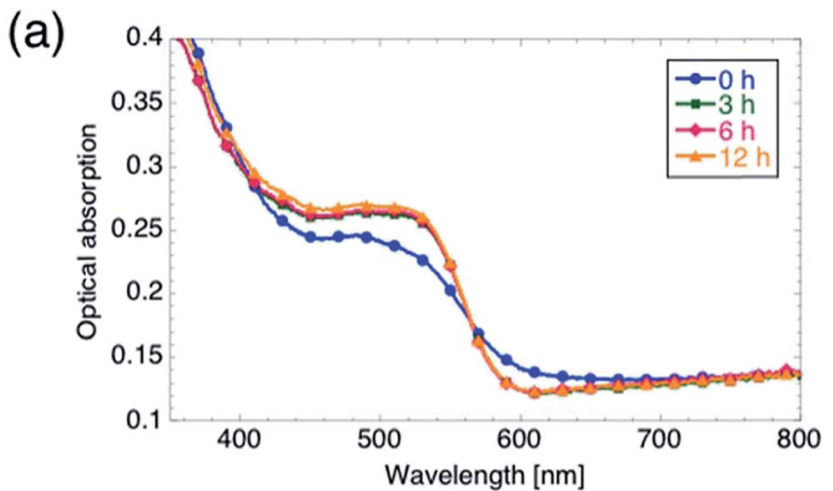

(b)

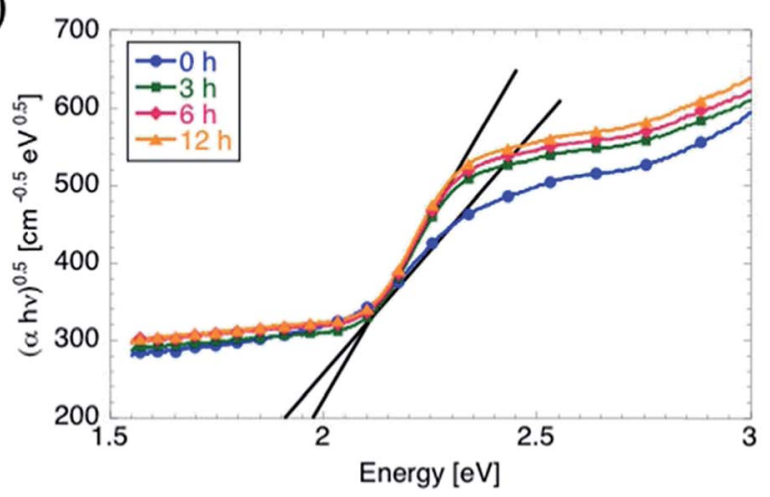

Fig. 5 Optical characterization of hematite films. (a) Optical absorption as function of wavelength. (b) Bandgap energy determination from Tauc analysis performed assuming an indirect bandgap in hematite. The bandgap energy $E_{\mathrm{G}}$ increases from 1.90 to $1.98 \mathrm{eV}$ between the $0 \mathrm{~h}$ sample and the other samples.

560 and $640 \mathrm{~nm}$. Inspection of the Tauc plots in Fig. 5b reveals that $E_{\mathrm{G}}$ increases from 1.9 to $1.98 \mathrm{eV}$ upon increasing $t_{\mathrm{ox}}$ from 0 to 3 hours, while further increasing $t_{\mathrm{ox}}$ leaves $E_{\mathrm{G}}$ unmodified. We believe, however, that the observed discrepancy in optical absorption between the $0 \mathrm{~h}$ samples and the other samples cannot account for the observed difference in $V_{\text {onset }}$, for the following reasons. First, an increase in number of absorbed photons (with no other changes in the electrochemical properties of the film) should lead to an increased $j_{\text {max }}$ rather than a shift in $V_{\text {onset }}{ }^{15}$ Second, the optical absorption is not affected by further increasing $t_{\text {ox }}$ from 3 hours to 6 or 12 hours, although $V_{\text {onset }}$ is shifted further cathodically. On the other hand, the slightly lower value of $j_{\max }$ measured on the $0 \mathrm{~h}$ sample as compared to the other samples (for which $j_{\max }$ is the same within a $5 \%$ margin) can be attributed to the lower optical absorption between 420 and $560 \mathrm{~nm}$ observed for the $0 \mathrm{~h}$ sample.

Complete conversion of Fe into hematite was confirmed in a previous work $^{56}$ by XRD measurements performed on samples at least 4 times thicker than those used here. It is thus reasonable to assume than the same holds for the ultra-thin films employed in this study. In spite of several attempts, we did not manage to obtain significant XRD data on these $25 \mathrm{~nm}$ thick films. Therefore, we resorted to TEM characterization in order to investigate the properties of the films in terms of crystallinity and grain boundaries. The results are

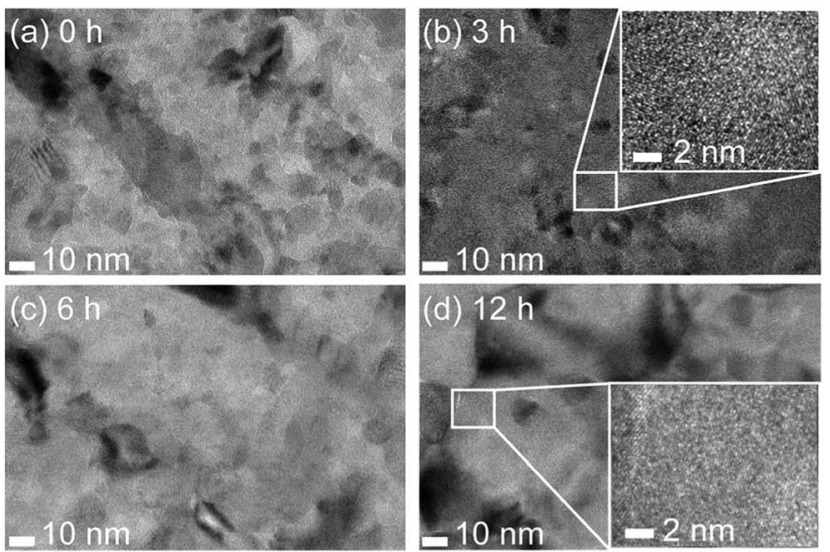

Fig. 6 TEM images of hematite films with $t_{\mathrm{ox}}=0 \mathrm{~h} \mathrm{(a)}, 3 \mathrm{~h}(\mathrm{~b}), 6 \mathrm{~h}$ (c) and $12 \mathrm{~h}(\mathrm{~d})$. Panels (b) and (d) also include insets showing high resolution images.

summarized in Fig. 6 . While all the films are polycrystalline as expected, we did observe clear changes in the sample at the nanoscale. In particular, the crystallite size increases distinctly with increasing $t_{\mathrm{ox}}$. For the lowest $t_{\mathrm{ox}}$, the average crystallite size is of the order of $10 \mathrm{~nm}$ or smaller. Since the film thickness is $25 \mathrm{~nm}$ there are several crystallites in the TEM field of view, which makes it somewhat difficult to interpret the images and in particular to perform quantitative analysis of the crystallite size distribution. Nonetheless, it is qualitatively clear that the crystallites grow with increasing $t_{\mathrm{ox}}$. For example, panel (d) $\left(t_{\mathrm{ox}}=12 \mathrm{~h}\right)$ shows crystallites that are significantly larger in size than the film thickness (several tens of $\mathrm{nm}$ ).

In view of the information obtained from the TEM analysis, we propose the following qualitative explanation for the shift of $V_{\mathrm{fb}}$, and therefore of $V_{\text {onset }}$. The increase of grain size for increasing $t_{\mathrm{ox}}$ determines a decrease in density of grain boundaries in hematite. These grain boundaries are typically associated with potential drops that reduce the efficiency of charge transport within the film. ${ }^{21}$ Therefore, by reducing the density of grain boundaries we also reduce the extent of the potential drops that are detrimental for the charge transport within hematite. In other words, a stronger band bending in hematite is obtained by increasing $t_{\mathrm{ox}}$. As a consequence, a larger potential is needed in order to reach the condition of zero band bending in hematite, which is $V_{\mathrm{fb}}$ by definition. Therefore, $V_{\mathrm{fb}}$ shifts towards lower, or towards more cathodic potentials. We observe that such mechanism is consistent with all data extracted from the EIS measurements, in particular with the decrease in bulk trapping resistance $R_{\text {trap }}$ recorded under illumination. Moreover, we highlight that the proposed mechanism for the shift of $V_{\mathrm{fb}}$, and thus of $V_{\text {onset }}$, is consistent with the similar values of $j_{\text {max }}$ measured at highly anodic applied potentials. In this regime, in fact, the mechanism limiting the photocurrent in hematite photoanodes is the number of photons absorbed, rather than the charge transport within the film or the OER kinetics at the surface. $^{15}$ 


\section{Conclusions}

In conclusion, in this work we systematically studied the correlation between the OER $V_{\text {onset }}$ and $V_{\mathrm{fb}}$ of hematite based photoelectrodes. We addressed the question if the OER onset potential $V_{\text {onset }}$ on hematite can be lowered by lowering the flat band potential $V_{\mathrm{fb}}$ of the material, and if this can be achieved without post-fabrication treatments. To this end, we employed a model system based on hematite ultra-thin films, and we determined how $V_{\text {onset }}$ and $V_{\mathrm{fb}}$ are affected by the oxidation time during fabrication of the photoanodes. We demonstrated a cathodic shift of $V_{\text {onset }}$ of $200 \mathrm{mV}$ on increasing $t_{\mathrm{ox}}$ from 0 to 12 hours, without any further surface treatment. A combination of AFM, XPS characterization and EIS measurements under illumination indicated that increasing $t_{\mathrm{ox}}$ did not affect the electrochemical properties of the hematite/electrolyte interface. EIS also pointed to increased charge transport efficiency within hematite for prolonged $t_{\mathrm{ox}}$. Mott-Schottky analysis revealed a quantitative correlation between the cathodic shift of $V_{\text {onset }}$ and a shift of $V_{\mathrm{fb}}$ in the same direction. The fact that the difference between $V_{\text {onset }}$ and $V_{\mathrm{fb}}$ is independent of $t_{\mathrm{ox}}$ offered further confirmation that varying the oxidation time did not affect the kinetics of the OER. From TEM analysis we proposed that the mechanism responsible for the shift of $V_{\mathrm{fb}}$ hematite, and therefore for the shift of the OER $V_{\text {onset }}$, is the improved quality of the films in terms of increased grain size and thus of reduced density of grain boundaries, leading to an increase in band bending in the semiconductor. The results presented here point at the possibility of enhancing the performance of hematite in terms of lowering $V_{\text {onset }}$ by improving the intrinsic properties of the semiconductor, which we expect to be compatible with further strategies towards the same goal such as introduction of underlayers, addition of electrocatalysts, or other surface coatings and post-fabrication treatments. Furthermore, the concepts presented in the Introduction regarding the energetics of a semiconductor/electrolyte interface can be applied not only to hematite, but much more generically to both n-type and p-type semiconductors. Therefore, the approach of shifting $V_{\mathrm{fb}}$ can readily be applied to any semiconductor-based photoelectrodes, i.e. not only lowering $V_{\mathrm{fb}}$ for n-type semiconductors used in oxidation half-cell reactions, but also increasing $V_{\mathrm{fb}}$ for p-type semiconductors employed for reduction half-cell reactions.

\section{Acknowledgements}

BI, BW, and IZ thank Formas (project numbers 219-2011-959 and 229-2009-772) for financial support. AH acknowledges support from the Swedish Research Council. BI thanks the Areas of Advance for Material Science and for Nanotechnology at Chalmers University of Technology supporting his external research stay at UNSW. Dr. Yunhau Ng and Prof. Rose Amal from the School of Chemical Engineering (UNSW) are gratefully acknowledged for providing the potentiostat for the PEC measurements performed at UNSW. GC and HZ acknowledge support from the Australian Government through the Australian Renewable Energy Agency (ARENA). The Australian
Government, through ARENA, is supporting Australian research and development in solar photovoltaic and solar thermal technologies to help solar power become cost competitive with other energy sources. The views expressed herein are not necessarily the views of the Australian Government, and the Australian Government does not accept responsibility for any information or advice contained herein.

\section{References}

1 M. Grätzel, Nature, 2001, 414, 338-344.

2 Y. Tachibana, L. Vayssieres and J. R. Durrant, Nat. Photonics, 2012, 6, 511-518.

3 T. Bak, J. Nowotny, M. Rekas and C. C. Sorrell, Int. J. Hydrogen Energy, 2010, 27, 991-1022.

4 A. Fujishima and K. Honda, Nature, 1972, 238, 37-38.

5 M. D. Hernández-Alonso, F. Fresno, S. Suárez and J. M. Coronado, Energy Environ. Sci., 2009, 2, 1231.

6 F. E. Osterloh, Chem. Mater., 2008, 20, 35-54.

7 Z. Li, W. Luo, M. Zhang, J. Feng and Z. Zou, Energy Environ. Sci., 2013, 6, 347.

8 B. Iandolo and A. Hellman, Angew. Chem., Int. Ed. Engl., 2014, 53, 13404-13408.

9 L. R. Yeh and N. Hackerman, J. Electrochem. Soc., 1977, 124, 833.

10 P. C. K. Vesborg and T. F. Jaramillo, RSC Adv., 2012, 2, 7933. 11 A. Murphy, P. Barnes, L. Randeniya, I. Plumb, I. Grey, M. Horne and J. Glasscock, Int. J. Hydrogen Energy, 2006, 31, 1999-2017.

12 J. Brillet, J. Yum, M. Cornuz, T. Hisatomi, R. Solarska, J. Augustynski, M. Graetzel and K. Sivula, Nat. Photonics, 2012, 6, 824-828.

13 L. A. Marusak, R. Messier and W. B. White, J. Phys. Chem. Solids, 1980, 41, 981-984.

14 L. M. Peter, J. Solid State Electrochem., 2012, 17, 315-326.

15 H. Dotan, K. Sivula, M. Grätzel, A. Rothschild and S. C. Warren, Energy Environ. Sci., 2011, 4, 958.

16 A. P. Singh, A. Mettenbörger, P. Golus and S. Mathur, Int. J. Hydrogen Energy, 2012, 37, 13983-13988.

17 G. Rahman and O.-S. Joo, Int. J. Hydrogen Energy, 2012, 37, 13989-13997.

18 R. Franking, L. Li, M. A. Lukowski, F. Meng, Y. Tan, R. J. Hamers and S. Jin, Energy Environ. Sci., 2013, 6, 500.

19 M. Barroso, S. R. Pendlebury, A. J. Cowan and J. R. Durrant, Chem. Sci., 2013, 4, 2724.

20 Photoelectrochemical Hydrogen Production, ed. R. van de Krol and M. Grätzel, Springer US, Boston, MA, 2012, vol. 102.

21 S. C. Warren, K. Voütchovsky, H. Dotan, C. M. Leroy, M. Cornuz, F. Stellacci, C. Hébert, A. Rothschild and M. Grätzel, Nat. Mater., 2013, 12, 842-849.

22 I. Cesar, K. Sivula and A. Kay, J. Phys. Chem. C, 2008, 772-782. 23 A. Kleiman-Shwarsctein, Y.-S. Hu, A. J. Forman, G. D. Stucky and E. W. McFarland, J. Phys. Chem. C, 2008, 112, 1590015907.

24 S. Kumari, A. P. Singh, D. Deva, R. Shrivastav, S. Dass and V. R. Satsangi, Int. J. Hydrogen Energy, 2010, 35, 3985-3990. 
25 A. Kay, I. Cesar and M. Graetzel, J. Am. Chem. Soc., 2006, 15714-15721.

26 Y. Lin, S. Zhou, S. W. Sheehan and D. Wang, J. Am. Chem. Soc., 2011, 133, 2398-2401.

27 A. Mao, G. Y. Han and J. H. Park, J. Mater. Chem., 2010, 20, 2247.

28 K. Sivula, F. Le Formal and M. Grätzel, ChemSusChem, 2011, 4, 432-449.

29 M. J. Katz, S. C. Riha, N. C. Jeong, A. B. F. Martinson, O. K. Farha and J. T. Hupp, Coord. Chem. Rev., 2012, 256, 2521-2529.

30 L. Badia-Bou, E. Mas-Marza, P. Rodenas, E. M. Barea, F. Fabregat-Santiago, S. Gimenez, E. Peris and J. Bisquert, J. Phys. Chem. C, 2013, 117, 3826-3833.

31 S. D. Tilley, M. Cornuz, K. Sivula and M. Grätzel, Angew. Chem., 2010, 122, 6549-6552.

32 F. Le Formal, K. Sivula and M. Grätzel, J. Phys. Chem. C, 2012, 116, 26707-26720.

33 F. Le Formal, N. Tétreault, M. Cornuz, T. Moehl, M. Grätzel and K. Sivula, Chem. Sci., 2011, 2, 737.

34 T. Hisatomi, F. Le Formal, M. Cornuz, J. Brillet, N. Tétreault, K. Sivula and M. Grätzel, Energy Environ. Sci., 2011, 4, 2512.

35 T. Hisatomi, H. Dotan, M. Stefik, K. Sivula, A. Rothschild, M. Grätzel and N. Mathews, Adv. Mater., 2012, 24, 26992702.

36 Y.-S. Hu, A. Kleiman-Shwarsctein, G. D. Stucky and E. W. McFarland, Chem. Commun., 2009, 2652-2654.

37 M. Zhang, W. Luo, N. Zhang, Z. Li, T. Yu and Z. Zou, Electrochem. Commun., 2012, 23, 41-43.

38 D. Cao, W. Luo, J. Feng, X. Zhao, Z. Li and Z. Zou, Energy Environ. Sci., 2014, 7, 752-759.
39 P. Hiralal, S. Saremi-Yarahmadi, B. C. Bayer, H. Wang, S. Hofmann, K. G. Upul Wijayantha and G. A. J. Amaratunga, Sol. Energy Mater. Sol. Cells, 2011, 95, 1819-1825.

40 B. Iandolo, T. J. Antosiewicz, A. Hellman and I. Zorić, Phys. Chem. Chem. Phys., 2013, 15, 4947-4954.

41 A. W. Grant, Q.-H. Hu and B. Kasemo, Nanotechnology, 2004, 15, 1175-1181.

42 R. Synowicki, Thin Solid Films, 1998, 313-314, 394-397.

43 B. Iandolo, B. Wickman, B. Seger, I. Chorkendorff, I. Zorić and A. Hellman, Phys. Chem. Chem. Phys., 2014, 16, 12711275.

44 F. Le Formal, M. Grätzel and K. Sivula, Adv. Funct. Mater., 2010, 20, 1099-1107.

45 B. Klahr, S. Gimenez, F. Fabregat-Santiago, T. Hamann and J. Bisquert, J. Am. Chem. Soc., 2012, 134, 4294-4302.

46 J. Bisquert, Phys. Chem. Chem. Phys., 2008, 10, 49-72.

47 Z. Chen, H. N. Dinh and E. Miller, Photoelectrochemical Water Splitting, Springer, New York, NY, 2013.

48 J. Schoonman, J. Electrochem. Soc., 1981, 128, 1154.

49 R. van de Krol, J. Electrochem. Soc., 1997, 144, 1723.

50 J. E. A. M. van den Meerakker, E. A. Meulenkamp and M. Scholten, J. Appl. Phys., 1993, 74, 3282.

51 J. A. Glasscock, P. R. F. Barnes, I. C. Plumb and N. Savvides, J. Phys. Chem. C, 2007, 111, 16477-16488.

52 B. Klahr, S. Gimenez, F. Fabregat-Santiago, J. Bisquert and T. W. Hamann, J. Am. Chem. Soc., 2012, 134, 16693-16700.

53 M. Tomkiewicz, J. Electrochem. Soc., 1979, 126, 1505.

54 M. Radecka, K. Zakrzewska, M. Wierzbicka, A. Gorzkowska and S. Komornicki, Solid State Ionics, 2003, 157, 379-386.

55 R. Beranek and H. Kisch, Electrochem. Commun., 2007, 9, 761-766.

56 B. Iandolo and M. Zäch, Aust. J. Chem., 2012, 65, 633. 